\title{
HealthCare Recommendation for Personalized Framework
}

\author{
Shreya B.Ahire \\ Department of Computer Enginnering \\ Marathwada Mitra Mandal's College of Enginnering \\ Pune
}

\begin{abstract}
Now a days, for any kind of information, people depend on internet. They use search engines like Google to search information over internet. The queries have to be accurate that will give the information related to user's Health Care.

But there is huge amount of information on the internet and so it's difficult to get the relevant information easily. In case of searches on right food exercise, people usually have their own preferences. Also people are restricted to some medical conditions so some foods and exercises are avoided so they are attracted towards other food and exercises. Also the civilization where people reside will have impact on the number of choices and varieties of food.
\end{abstract}

So there is need for easy to use framework for food and exercise recommendation. We propose ontology based framework for Health Care recommendation system which will provide precise information based on users requirements and constraints. This framework will use semantic web technology to analyze user's preferences and will build a nourished and health associated use's profile and will use the profile to categorize the associated knowledge so that users can make delicious food and exercise inquiries. We will also use the Decision Tree algorithm for retrieving related information from the database.

\section{General Terms}

Data mining and Information Retrieval, Decision tree.

\section{Keywords}

Personalization; food and nutrition; exercise; Health Care; ontology; decision tree

\section{INTRODUCTION}

This is the age of Computers where internet is used in world wide. In which web information grows tremendously as the amount of information available on the internet has raised significantly and this makes getting the relevant information is more challenging as its get difficult to search correct information on the internet. Many people are interested in the appropriate and meaningful information from the web. The web search engines has some constraint which provide adequate information to many people and some are not interested because the output of information is not relevant on the web to their needs[1]. People searches the information on the popular sites from which they can get and collect relevant and precise information on the food, health and nutrition. Many people searches on popular social sites where the information available is on the bases of the keywords and this does not satisfied the users need. The search engine should view the information as per the user's needs as they can get relevant information. This is very important in health and

\author{
Harmeet Kaur Khanuja \\ Department of Computer Enginnering \\ Marathwada Mitra Mandal's College of Enginnering \\ Pune
}

nutrition area when some food are restricted to user's because of their health conditions[1].

As people need some amount of nutrition to preserve the healthiness even though people are careless about their daily nutrition. Now a days the working styles and the development of unhealthy life habits have been reduced perceived importance of exercise, and experts increasingly suggest that physical inactivity will be the most important health problem of the twenty-first century[4]. Health care policy are driving an increased focus on costs, quality and transparency of care[10]. This new focus on improving the quality and efficiency on health care.

In this paper, we will use framework for food and exercise recommendation. We propose an ontology based semantic Health Care recommendation system which will provide precise information based on users requirement and constraints. This framework will use the technology to analyze user's preference and will build a nourished and health associated user's profile and will use the profile to categorize the associated knowledge so that users can make delicious food and exercise inquiries.[1]. We will also use the Decision Tree algorithm for retrieving related information from the database.

A semantic framework for food recommendation based on the user's preferences, as the people have their own choices of food, the health condition may vary from people to people so the food choices would also differ. So this will be better way to have health and food related queries will help in providing to give relevant information to user personalized choices of food and nutrition from popular food and health sources. We use the semantic processing techniques help in(a) better understanding the user's queries and(b) better structuring the scattered information on the Web. This would provide to get more relevant and better result. So, we use the personalization technologies which help in recommending more relevant information to the user's needs[1].

\section{MOTIVATION AND CHALLENGES}

The information on the internet has become challenging issue for users. The user need to be clear about their needs to get correct information.

The amount of web-base information available on the internet has become the most challenging job today's scenario. People are interested in relevant and interested information from the web. There is huge amount of information on the internet so it's difficult to get relevant information related to users Health Care and for this user needs more intelligent system(agents) to gather the useful information. In case of searches on right food and exercise, people usually have their own preferences. Also people are restricted to some medical conditions so some foods and exercises are avoided so they are attracted towards 
other food and exercises. Also the civilization where people reside will have impact on the number of choices and varieties of food. So there is need for easy to use frame work for food and exercise recommendation.

We propose an ontology based semantic framework Health care recommendation system which will give relevant information to the user's needs and constraints. This framework is to analyze user's preference and will and will build a nourished and health associated user's profile and will use the profile to categorize the associated knowledge so that users can make delicious food and exercise inquiries.[1].

\section{LITERATURE SURVEY}

Ahmed Al-Nazer et al.[1]2014,proposed semantic Web and ontology techniques to interpret user's taste, build a nourished and health associated user's profile, and use the profile to categorize the associated knowledge so that users can make delicious food and exercise inquiries. A semantic framework that uses the personalization techniques based on integrated domain ontologies, pre-constructed by domain experts, to recommend the relevant food that is consistent with people's needs. The empirical evaluation of the proposed framework shows promising results for recommending the relevant food information with superior user's satisfaction.

X. Tao et al.[2] 2011,proposes a personalized ontology model is proposed for knowledge representation and reasoning over user profiles. This model learns ontological user profiles from both a world knowledge base and user local instance repositories. The ontology model is evaluated by comparing it against benchmark models in the web information gathering. The results show that this ontology model is successful.

Jen-Heo et al.[3]2010,proposed personalized diet planning approach not only translates nutrient recommendation into realistic dish choices, but also accepts feedbacks from users to fine-tune their meal plans. The results showed that daily nutrition needs can fulfilled by the designated meals, and the interactive diet planning scheme helps a user adjust the plan in an easier way. The guidelines generated by SmartDiet are expected to potentially improve the overall health and reduce the risk of chronic diseases of individuals.

J.Chiang et al.[4]2014,in this paper a portable activity pattern recognition system designed to automatically recognize the daily activity habits of users, and provide visualized life logs on the wellness self-management platform for patients and clinicians. Based on the participants and clinician's comments, appropriate modifications were made.

Irini Genitsaridi et al.[5]2013,proposes an evaluation study on PHR systems that provides an insight on their current status with regard to functional and technical capabilities and extensions to a specific PHR system. A requirement analysis that formulates the composite evaluation model which use to perform a systems review on numerous available solutions.

\section{SYSTEM ARCHITECTURE}

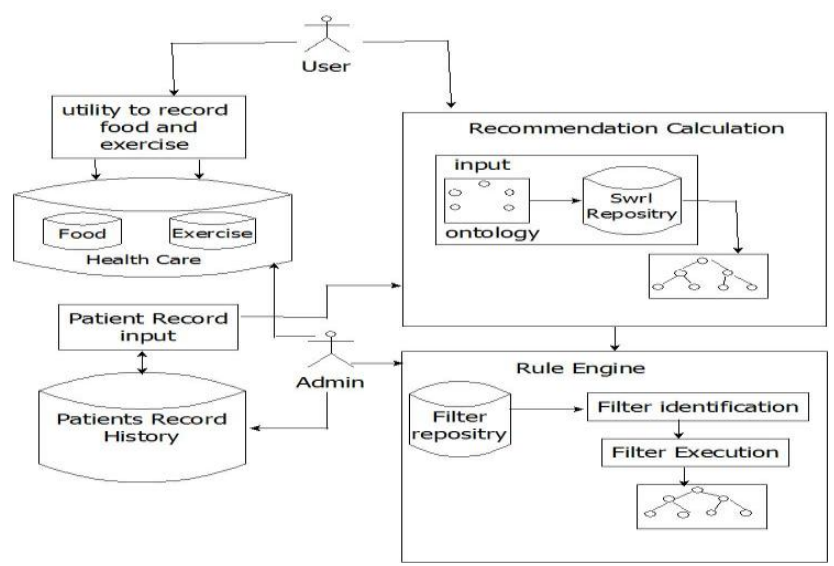

Fig.1 Health Care Recommendation for Personalized Framework

In Fig.1.an application of users profile which contain the HealthCare preferences and the likes and dislikes of the food as it is needed for personalizing recommendations such as food, exercises, health condition. From users profile the application will maintain the history of the users and keeps the record of the information into the databases. Whenever user will search for relevant data related to health the preferred food and exercise would be recommended to the user by searching the health condition from the database that the user would recommend to the user by searching the health condition from the database that the user has previous any health related problems.

Database is needed to maintain the record of the users profile based on Health Care information. This is useful when the user searches for relevant diet and exercise this is easy to recognize from the database that what is good to recommend the user requirement by searching the health condition. According to the food categories user can select the preferred food category which the user want; also the application would recommend the other related food with having nutrition value to the users.

People searches for food and exercise for health condition. The application recommends food and an exercise to the users health condition. Also we will recommend the exercise through the users categories. As exercise and physical activity are more important to health and the prevention and treatment of many chronic diseases.

In the recommendation Calculation all the information of the patient will be maintain from the information user profile that user has entered. Also the history of the user is maintain regarding about health issue, food and exercise. The recommendation calculation is done by using ontology, Swrl(semantic web rule language) and decision tree. Ontology handles the rule based generation using decision tree with respect to the current status of the user e.g. age, health related problems. Ontology is used to construct decision tree support where it is used to define properties, attributes and restrictions.

In the rule engine filters repository is the database that is used for number of condition that are based on medical conditions and dietary rules. Filters identification is used for what type of data or query the users have entered; according to the condition the execution will be done then it will generate the 
reports that will recommend the diet, nutrition and exercise to the user.

\subsection{Algorithm}

The tremendous rise in the technological development in the healthcare systems has to be maintain history through variety of methods. The Decision tree algorithm is one of the method used in healthcare systems.

Decision tree is an interactive application, which is developed to support physicians, and healthcare professionals for decision making[8]. To analyze the characteristics of users the decision tree can provide the relevant information to users by recommendation, follow-up, and monitoring.

Here, we propose a simple algorithm steps for Healthcare recommendation

1.Start the process

2.Input User/Patient Name and his current medical condition

3.Search for the User/patient for his history.

i. If Found, get his all major medical conditions stored in the history tables.

4. Calculate the food recommendation using a decision tree based on:

i. Subset of Allowed food items for all of the medical conditions.

ii. Daily requirement of the Nutrition based on the age of the patient.

iii. Food preferences of the User/patient, likes and dislikes

iv. His daily nutritional needs based on age and weight
v. His economical status
vi. Food allergies

5. Calculate the exercise recommendations using decision tree based on:
i. Medical condition
ii. Age/ weight
iii. Allergies
iv. Preferences
v. Life style

6. Display the result of Food recommendation and Exercise recommendations calculate above.

7. Update the patient history with the current medical conditions and the recommendations provided.

\section{Stop process.}

\section{CONCLUSION}

In this paper, we used a framework for food and exercise recommendation. We propose an ontology based semantic framework Health Care recommendation system which will provide precise information based on users requirement and constraints. This framework will use semantic web technology to analyze user's preferences and will build a nourished and health associated user's profile and will use the profile to categorize the associated knowledge so that users can make delicious food and exercise inquiries. As the framework is fully automated which gives relevant information to users profile with less intervention from domain experts.

The application and techniques will be efficient, feasible and user friendly that would be less time consuming. In future work, we will on more enhancement and gather more feedback from users and also we can connect to the healthcare centers, which will allow healthcare providers that will assist the users who have medical problem.

\section{ACKNOWLEDGMENTS}

We would like to acknowledge Prof. Ram Joshi for his valuable comments and opinion about the dissertation work also we received both direct and indirect support from my teachers, friends, and family.

\section{REFERENCES}

[1] Ahmed Al-Nazer, Tarek Helmy, Mohammed AlMulhem," User's Profile Ontology-Based Semantic Framework for Personalized Food and Nutrition Recommendetion",Elsevier, Procedia Computer Science, 2014.

[2] Xiaohui Tao, Yuefeng Li, Ning Zhong," A Personalized Ontology Model for Web Information Gathering",IEEE transcations on knowledge and data engineering,vol.23,no.4, April 2011.

[3] Jen-Hao Hsiao, Henry Chang,"SmartDiet: A Personal Diet Consultant for Healthy Meal Planning,"IEEE $23^{\text {rd }}$ International Conference, Computer Based Medical System(CBMS),2010.

[4] Jung- Hsien Chiang, Pei-Ching Yang,Hsuan Tu,"Pattern analysis in daily physical activity data for personal health management",Elsevier, Pervasive and Mobile Computing,2014.

[5] Irini Genitdaridi,Haridimos Kondylakis,Lefteris Koumakis,Kostas Marias,Manolois Tsiknakis",Towards IntellegentPersonal Health Record system:review,criteria and extensions,"Elsevier,Procedia computer science,2013

[6] Abdullah A. Aljumah, Mohammed Gulam Ahamad, Mohammad Khubeb Siddiqui", Application of data mining: Diabetes health care in young and old patients," Production and hosting by Elsevier B.V. on behalf of King Saud University,2013

[7] Chrystalleni Lazarou, Minas Karaolis , Antonia-Leda Matalas, Demosthenes B. Panagiotakos," Dietary patterns analysis using data mining method. An application to data from the CYKIDS study", Elsevier, Computer Methods and Program in Biomedicine,2012

[8] Muthuraman Thangaraj, Sankaran Gnanambal," A Rule Based Decision Support System for Aiding Vitamin D Deficiency Management",Indian Journal of Science and Technology, Vol 7(1), 48-52, January 2014

[9] Thangaraj Muthuraman, Gnanambal Sankaran," A Framework for Personalized Decision Support System for the Healthcare Application", Health Science Journal,Vol8(2),2014

[10] Kelly A. Tappenden,Beth Quatrara,Melissa L. Parkhurst, Ainsley M.Malone,Gary Fanjiang, Thomas R. Ziegler," Critical Role of Nutrition in Improving Quality of Care: An Interdisciplinary Call to Action to Address Adult Hospital Malnutrition",Journal of the Academy of Nutrition and Dietetics, 17 July 2013 